\title{
Channel Estimation in OFDM System over Fading Channels: A Compressive Sensing based Approach
}

\author{
Ashish Jha and Lavish Kansal* \\ School of Electronics and Electrical Engineering, Lovely Professional University, Phagwara - 144411, Punjab, India; \\ jha.ashish256256@gmail.com, lavish.15911@lpu.co.in
}

\begin{abstract}
As Least Square (LS) and Minimum Mean Square Error (MMSE) do not give the satisfactory result to justify the features of the wireless channel, Compressed Sensing (CS) based on Discrete Sine Transform (DST) for channel impulse response has been studied which gained much more importance in digital signal processing. We have used Compressed Sensing to estimate the channel coefficients of the fading channel; then we have performed the CS recovery algorithm to estimate the channel and to nullify the fading effect, the thus much better result are obtained in the simulation which satisfies the better performance of the system as compared to the traditional method. Simulation results show a considerable improvement in Bit Error Rate (BER) performance on employing the Compressed Sensing approach in comparison to LS and MMSE. The improvement of the order of 2-9 dB of SNR is for a particular value of BER.
\end{abstract}

Keywords: Channel Estimation, Compressed Sensing, DST, LS, MMSE, OFDM

\section{Introduction}

The principle behind the system is to allocate the available frequency into several sub-carriers, then again converting a frequency selected channel into a parallel connection frequency at sub-channels. To obtain high spectral efficiency, different spectra overlap with the sub-carrier frequency and have minimum frequency separation to maintain orthogonality of their waveforms. $\mathrm{CP}$ is used to maintain orthogonality and eliminate ISI between two symbols. When the information bits are collected they are firstly coded and then modulated, they are fed into N-point Inverse Fast Fourier Transform (IFFT) ${ }^{4}$. Following IFFT block, cyclic extension is done to remove inter-symbol or inter-carrier interference. The digital to analog converter provides sampling period to an Orthogonal Frequency Division Multiplexing (OFDM), which has the bandwidth of $1 /$ Ts, where Ts is described as sampling interval. The channel is known for its impulse response followed by Additive White Gaussian Noise (AWGN) noise. In the receiver side (A/D) is done and the Cyclic Prefix (CP) is removed, the N-point FFT is used to change the data back to the frequency domain, at last, the information bits are recovered after the channel equalisation, further demodulation ${ }^{1-4}$.

The performance of the system is mostly defined by the channel estimation techniques used to nullify the channel effect. One can achieve that performance by either pilot design methods or the channel estimation methods. Many of the channel estimation schemes were proposed in earlier to improve the system performance in wireless communication 2 . LS algorithm being the simplest having great noise content and have very less calculation complexity ${ }^{2}$. MMSE shows better performance than the LS estimator but has much more calculation complexity. This is practically not possible to implement as each time we need matrix inversion and Channel State Information (CSI). Wireless channel shows sparse property having very less impulse response non-zero. LS and MMSE do not consider sparse property into account $\stackrel{3}{*}$.

In recent world, CS theory has taken much more importance and claims that signal can be recovered exactly with the sampled signal at a sampling rate below Nyquist rate if the present signal holds sparse prop-

${ }^{*}$ Author for correspondence 
erty ${ }^{4}$. Keeping this function into account CS theory has been implemented in channel estimating of the wireless channel. The optimization techniques for orthogonally matching pursuit to increase the precision of the algorithm based on channel characteristics is given in ${ }^{5}$. The novel optimization method for OMP, which requires one iteration with the channel information extracted by the LS estimation, is presented 6 . A subspace pursuit to reconstruct the signal based on compressed sampling is proposed $^{\mathrm{T}}$. Signal recovery from random measurement by OMP techniques is presented $\stackrel{8}{*}$. Sparse techniques used for multipath fading in the wireless channel have been given ${ }^{9}$ which show modeling and implication of the multipath channels. Optimized pilot placement for the multipath channel in OFDM system is implemented by ${ }^{10}$ which focused on improvement of system performance. In this paper, the focus is to assess the performance of OFDM system by employing new channel estimation techniques Compressed Sensing based on DST. In the next section, we describe the various kinds of techniques in CS, different types of channels estimation schemes and comparison between LS, MMSE and CS techniques. Later sections comprise analysis of results and finally the conclusion.

\section{Model Description}

We have implemented various channel estimation schemes for the OFDM system which are discussed in the further section of the paper. The basic model to represent the wireless communication system is shown in Figure 1.

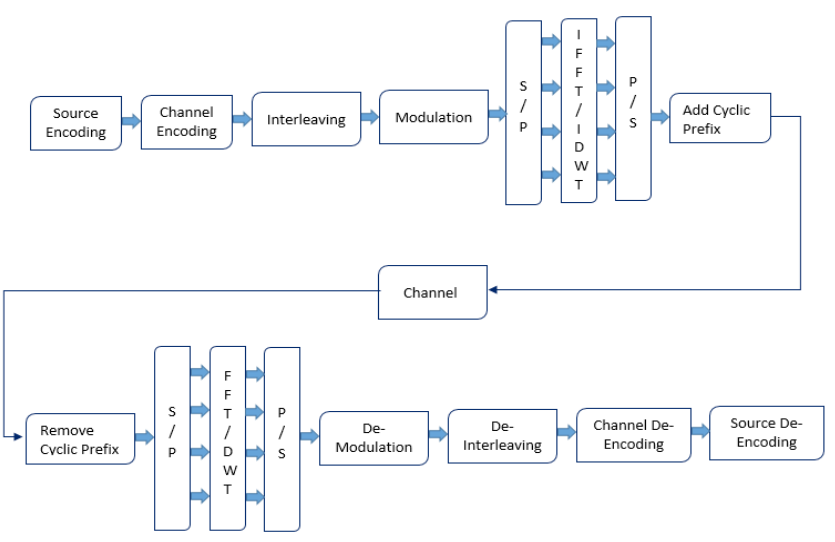

Figure 1. OFDM system model.

The property of signals that are mutually independent of each other is called as orthogonality ${ }^{1}$. In OFDM each sub carrier is well spaced out to maintain orthogonality and each carrier are modulated with individual frequency which can be shown as:

$$
\int_{0}^{T}[\cos (2 \Pi f n t)+\cos (2 \Pi f m t) d t=\delta(\operatorname{\Pi n} n-m)
$$

Where, $\delta(n-m)$ is the Dirac Delta Function.

The sub carrier frequency $f_{n}$ is defined as:

$f_{n}=n \Delta f$

$\Delta f=\frac{f_{s}}{N}=\frac{1}{N T}$

Here, $f_{s}$ is used to denote entire bandwidth and $N$ is the number of sub carriers.

\subsection{Least Square Estimation}

OFDM channel estimation symbols will be transferred periodically in block-type pilot-based channel estimation. In this type based on the pilot signals and received signals estimating the channel conditions will be done. The receiver uses the estimated channel condition to decode the data inside the block. The least-square estimator minimizes the parameter:

$(\bar{Y}-\underline{X} \bar{H})^{H}(\bar{Y}-\underline{X} \bar{H})$,

Where,

$(.)^{\mathrm{H}}$ - Conjugate transpose operation.

$\bar{Y}$ - received signals.

$\underline{X}-$ Pilot signal specified by matrix.

Then, the LS estimator of $\bar{H}$ is given by:

$$
\begin{aligned}
& \left.\hat{H}_{L S}=\underline{X}^{-1} \bar{Y}=\left[-\left(X^{-}\right]_{k} / Y_{k}\right)\right]^{T} \\
& (\mathrm{k}=0, \ldots, \mathrm{N}-1)
\end{aligned}
$$

The LS estimator can calculate the channel conditions even without having any knowledge about the statistics of the channel with very low complexity for calculation. But these calculations are suffering from high mean square error.

\subsection{MMSE Estimation}

The MMSE method minimizes the Mean Square Error by employing second - order statistics of the channel conditions ${ }^{9}$. Assuming that the channel vector $\bar{g}$ and the noise $\bar{N}$ are uncorrelated, it is derived that:

$$
R H H=E\{H H H\}=F R g g F H
$$




$$
R g Y=E\{0=R\}=R g g, F H X H
$$

$R Y Y=E\{\square \square H\}$

$=X F R g g F H X H+\sigma 2 N I N$

$\underline{R}_{g g}, \underline{R}_{H H}, \underline{R}_{Y Y}$ - Represents auto-covariance matrix of $\bar{g}, \bar{H}, \quad \bar{Y}$ respectively.

$\underline{R}_{g Y}$ - Represents the cross-covariance matrix between $\bar{g}$ and $\bar{Y}$.

\section{$\sigma_{N}^{2}-$ Represents the noise variance.}

By assuming that the auto-covariance matrix and noise variance are known at the receiver in advance, and the MMSE estimator is of $\bar{g}$ is given by $\hat{g}_{M M S} z$ may not be a Minimum Mean Square Error. At last, it is calculated that:

$$
\begin{aligned}
& \hat{\mathrm{g}}_{\text {mmse }}=\mathrm{R}_{\mathrm{gY}}{ }^{-1} \mathrm{Y}^{\mathrm{HH}} \\
& \hat{\mathrm{H}}_{\text {mmse }}=\mathrm{F} \hat{\mathrm{g}}_{\text {mmse }}
\end{aligned}
$$

The MMSE estimator surely yields much better performance than the LS estimator, but the only major drawback is its high computational complexity.

\section{Compressed Sensing}

The CS-based approach defines the problem of reconstructing the $\mathrm{N} \times 1$ signal from the small portion of the linear management. Consider an $\mathrm{N} \times 1$ vector $\mathrm{x}$, which can be represented as in some orthogonal basis $\psi=[\psi 1, \psi 2, \psi 3, \ldots \ldots \ldots, \psi \mathrm{N}]$.

$$
x=\psi \Theta
$$

? is a K-sparse representation of $\mathrm{x}$, where $\mathrm{k}<<\mathrm{N}$ because only $\mathrm{k}$ coefficient of ? are not zero. From CS theory, $\mathrm{x}$ can be recovery from a linear measurement vector:

$$
y=¥ \Theta \psi=¥ x
$$

Where $¥$ is an $\mathrm{M} \times \mathrm{N}(\mathrm{M}<\mathrm{N})$ observation matrix satisfying Restricted Isometric Property (RIP). Restricted Isometric Property: The observation matrix 0 is said to satisfy the Restricted Isometric Property of order S with parameter $\square_{s} \in(0,1)$, if:

$$
\mathrm{C}\left(1-\gamma_{s}\right)|| z|| 2 l 2<=\left(1+\gamma_{s}\right)|| z|| 2 l 2
$$

Holds for all S-sparse vectors $\mathrm{z} \in \mathrm{R}^{\mathrm{n}}$. If there is no noise in observation $y$, the reconstruction problem can be solved by $l_{1}$ norm optimization. Basis Pursuit (BP) is appropriate to solve the basis pursuit problem. If the observation $y$ is contaminated with noise, then an additional norm of the residual $\phi \psi o-y$ should be minimized such that:

$$
y=\phi \psi \emptyset
$$

Here we have used CS in DST domain. To create the DST matrix we have used the following equations which are shown below:

DST Matrix

$$
\begin{aligned}
& y(k)=\sum_{n=1}^{N}\left[x(n) \sin \left(\pi \frac{k n}{N+1}\right]\right) \\
& \text { where } \mathrm{k}=1 \text { to } \mathrm{N} \\
& \text { incr }{ }^{n}{ }^{n+\cdots} \\
& \frac{x(k) 2}{N+1} \sum_{n=1}^{N} y(n) \sin \left(k n \frac{\pi}{N+1}\right) \\
& \text { where k=1 to } \mathrm{N}
\end{aligned}
$$

\section{Rayleigh and Rician Channel}

The performance of wireless communication is mainly governed by wireless channel environment. The wireless channel should be dynamic and unpredictable which make the exact analysis of wireless channel. When there are multiple indirect paths between the source and destination, the Rayleigh fading occurs. The Rayleigh fading will scatter, diffract or reflect the signal. It is considered as the specialized form for stochastic fading. The Rayleigh distribution is used to characterize the amplitude gain of Rayleigh fading. It is mainly used when there is no sight of the line between transmitters and receivers and where many buildings and objects will attenuate, reflect and refract the signal.

When there is a presence of the strong dominant component, then the model must follow Rician distribution. The fading of Rician and Rayleigh is similar to one another except the presence of strong dominant in Rician fading $^{1-3}$. When one of the paths having the line of sight greater than the other the Rician fading will occur. The Rician fading will be expressed as:

$$
f_{x}(x)=\frac{x}{\sigma^{2}} e^{-\frac{x^{2}+c^{2}}{2 \sigma^{2}} l_{e}\left(\frac{X C}{\sigma^{2}}\right)}
$$

Where:

$\mathrm{I}_{0}=$ Modified Bessel function of order 0.

$\mathrm{C}=$ strength of the direct component. 


\section{Results and Discussion}

Here we have given the comparison between LS, MMSE and CS techniques. Thus, performance of the each estimator is checked with no channel estimation as the reference. In CS techniques, we have recovered the signal from the sampled version at a much-bellowed sampling rate. The transform used here for the compression is DST. The comparison is shown below for different channel as given in Table 1 . We have given the simulation results to compare the performance of the proposed LS, CS and MMSE techniques both at Rayleigh and Rician channel for OFDM system. The data sequences are modulated by M-PSK modulation. The Compressed Sensing techniques use DST transform for compressing the data thus the performance is evaluated at a particular channel. Figure 2 (a-h) shows the SER and SNR curve with LS, MMSE, CS and NO channel with the present simulation we observe that LS estimation is very prone to AWGN noise but have low complexity. CS estimation shows the better result as compared to LS. MMSE shows the best result but in practical, it is very difficult to know the channel statistics in advance. Here all these estimation schemes are done over Rayleigh channel thus for each modulation schemes graph has been shown below from Figure $2(\mathrm{a}-\mathrm{h})$. At higher modulation, it's become difficult to recover the signal from sparse property thus the performance of the CS based estimator deteriorates this is shown in Figure 2 (f-h). We observe that SNR required to achieve the SER up to $10^{\wedge}(-3)$ for different estimation schemes at Rayleigh channel are: For LS estimation it takes about $25 \mathrm{~dB}$ while for MMSE it takes only $18 \mathrm{~dB}$. CS require $16 \mathrm{~dB}$ and for no channel condition, it takes only $12 \mathrm{~dB}$ to achieve the

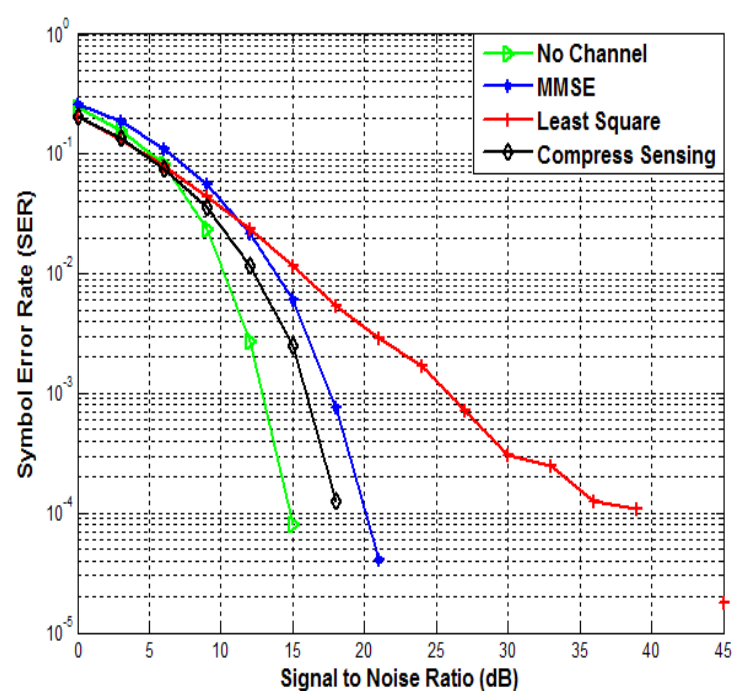

(a)

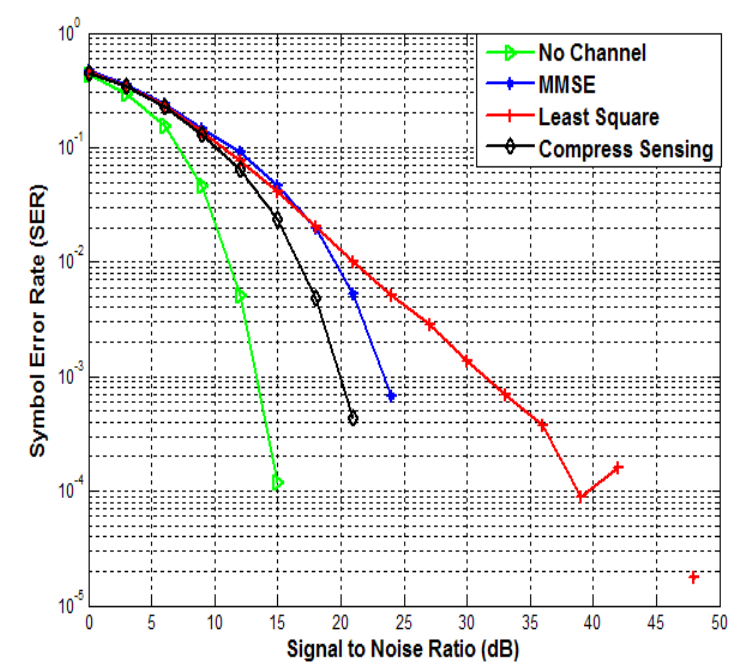

(b)

Table 1. BER performance in Rayleigh and Rician channel

\begin{tabular}{|l|l|l|l|l|l|l|l|l|}
\hline \multirow{3}{*}{ M-PSK } & \multicolumn{9}{|l|}{ SNR VALUE TO ACHIEVE BER UPTO 10^(-3) } \\
\cline { 2 - 10 } & \multicolumn{6}{|l|}{ RAYLEIGH Channel } & \multicolumn{4}{l|}{ RICIAN Channel } \\
\cline { 2 - 10 } & $\begin{array}{l}\text { NO } \\
\text { Channel }\end{array}$ & LS & MMSE & CS & $\begin{array}{l}\text { NO } \\
\text { Channel }\end{array}$ & LS & MMSE & CS \\
\hline 2 & 12 & 25 & 18 & 16 & 13 & 25 & 16 & 17 \\
\hline 4 & 14 & 30 & 24 & 20 & 14 & 27 & 15 & 20 \\
\hline 8 & 17 & 35 & 20 & 25 & 17 & 37 & 20 & 25 \\
\hline 16 & 22 & 42 & 34 & 33 & 22 & 42 & 27 & 32 \\
\hline 32 & 26 & 46 & 37 & 43 & 26 & 46 & 30 & 43 \\
\hline 64 & 33 & $>45$ & 35 & $>45$ & 32 & $>45$ & 37 & $>45$ \\
\hline 128 & 38 & $>45$ & $>45$ & $>45$ & 37 & $>45$ & 40 & $>45$ \\
\hline 256 & 42 & $>45$ & $>45$ & $>45$ & 43 & $>45$ & $>45$ & $>45$ \\
\hline
\end{tabular}




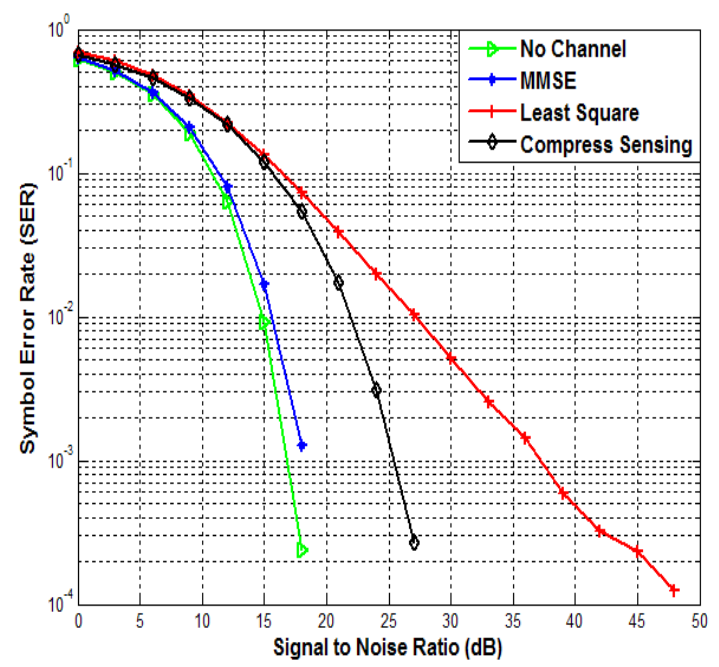

(c)

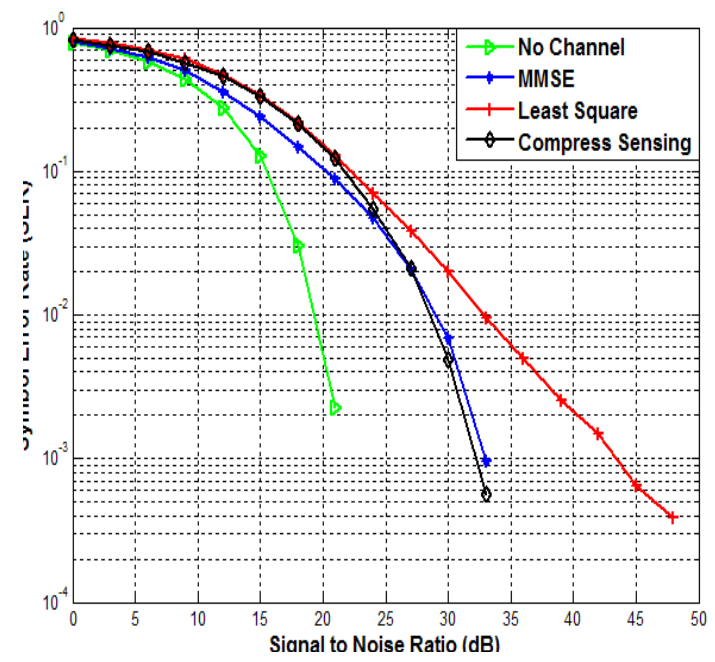

(d)

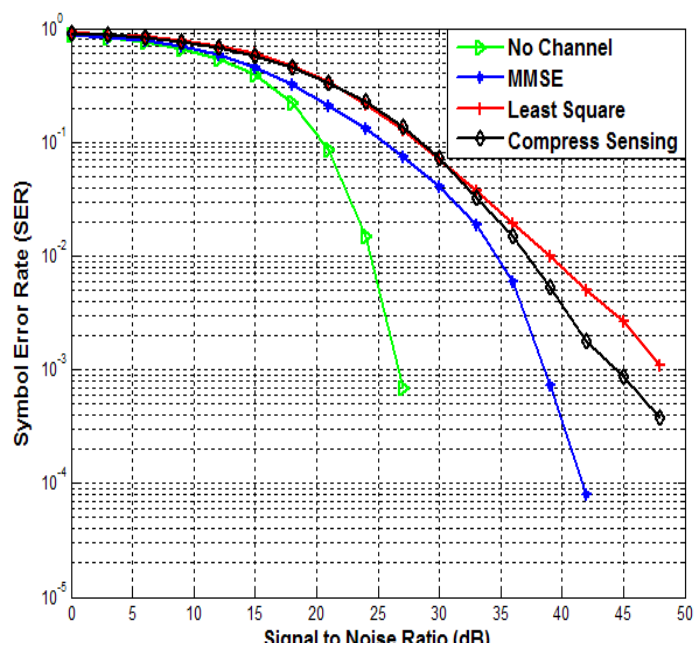

(e)

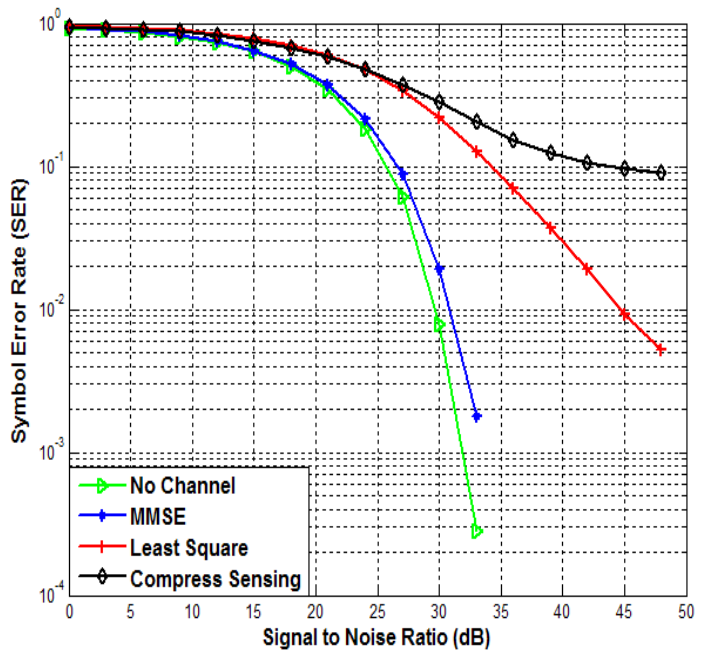

(f)

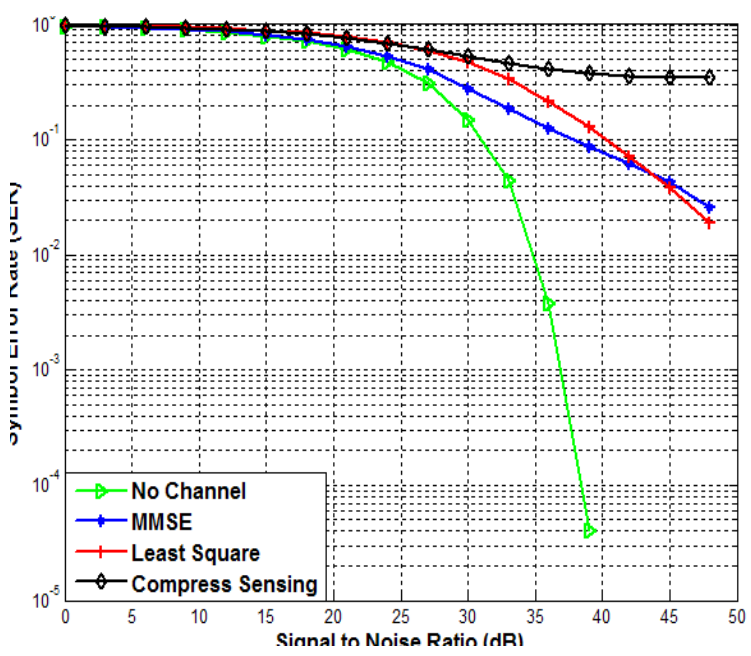

(g)

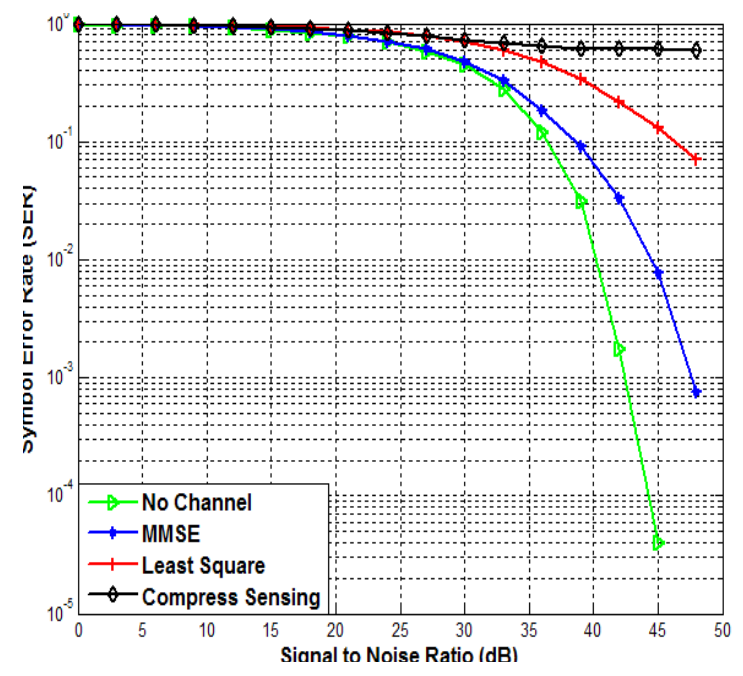

(h)

Figure 2 (a-h): SER vs SNR comparison for LS, MMSE and CS-DST estimating technique in Rayleigh Channel (a) BPSK (b) QPSK (c) 8-PSK (d) 16-PSK (e) 32-PSK (f) 64-PSK (g) 128-PSK (h) 256-PSK. 
same for BPSK modulation. Similarly, we observed for QPSK modulation that SNR required for LS estimation is $30 \mathrm{~dB}$, MMSE is $24 \mathrm{~dB}$ while for no channel it is 14 $\mathrm{dB}$ and CS required $20 \mathrm{~dB}$ to achieve that SER. Similarly, this is also observed for higher order modulation to validate the estimators' performance. This means CS based required much more attention at higher modulation and delay calculation.

Figure $3(\mathrm{a}-\mathrm{h})$ shows the SER and SNR response of the various estimation schemes over the Rician channel. MMSE being the best, CS outperform the LS estimation showing the better response. Over Rician channel all the three estimators are compared with no channel response and are shown in Figure 3 (a-h). At higher modulation, it's become difficult to recover the signal from sparse property thus the performance of the CS based estimator deteriorates this is shown in Figure 3 (f-h). We observe that SNR required achieving the SER up to $10^{\wedge}(-3)$ for different estimation schemes at Rician channel are: For LS estimation it takes about $25 \mathrm{~dB}$ while for MMSE it takes only $16 \mathrm{~dB}$. CS requires $17 \mathrm{~dB}$ and for no channel condition, it takes only $13 \mathrm{~dB}$ to achieve the same for BPSK modulation. Similarly, we observed for QPSK modulation that SNR required for LS estimation is $27 \mathrm{~dB}$, MMSE is $15 \mathrm{~dB}$ while for no channel it is $14 \mathrm{~dB}$ and $\mathrm{CS}$ required $20 \mathrm{~dB}$ to achieve that SER. Similarly, this is also observed for higher order modulation to validate the estimators' performance. This means CS based required much more attention at higher modulation and delay calculation.

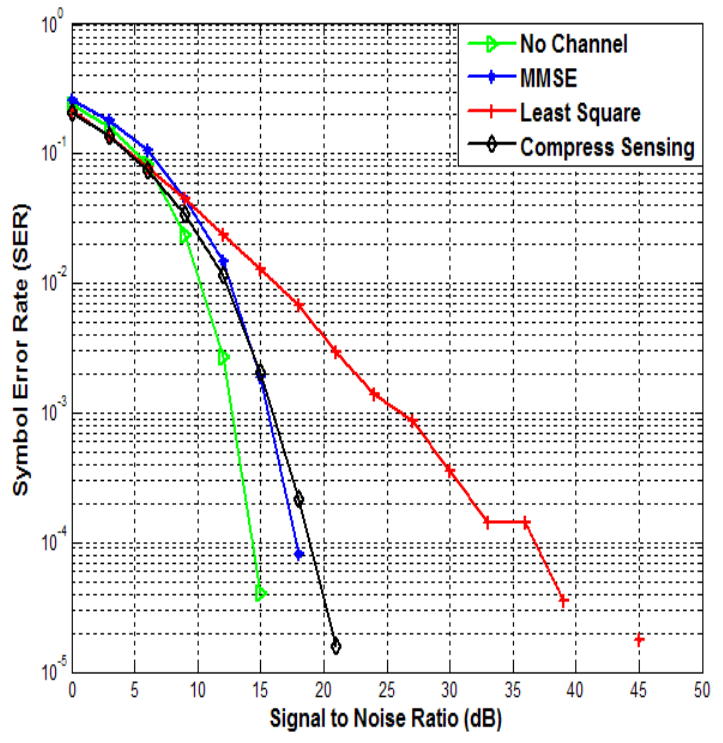

(a)

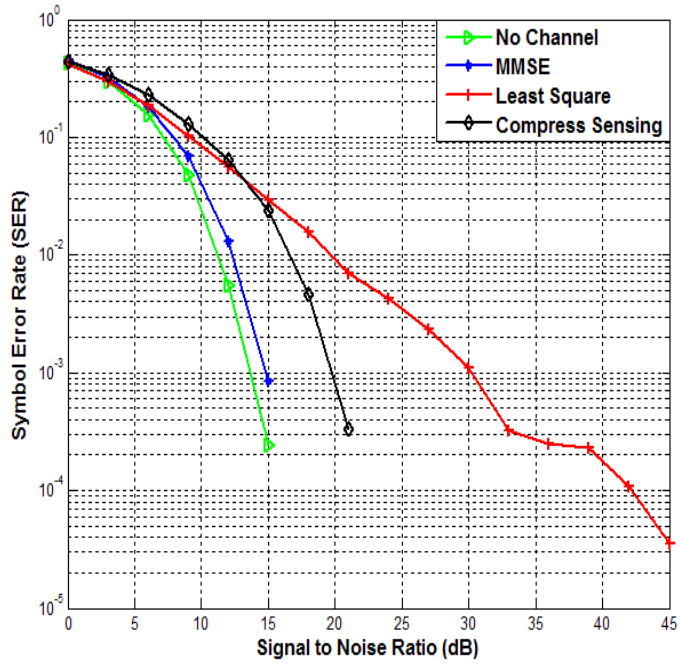

(b)

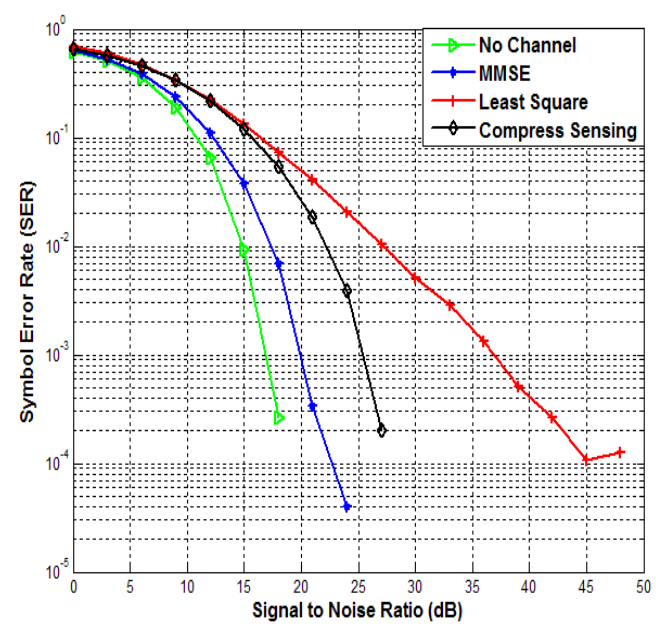

(c)

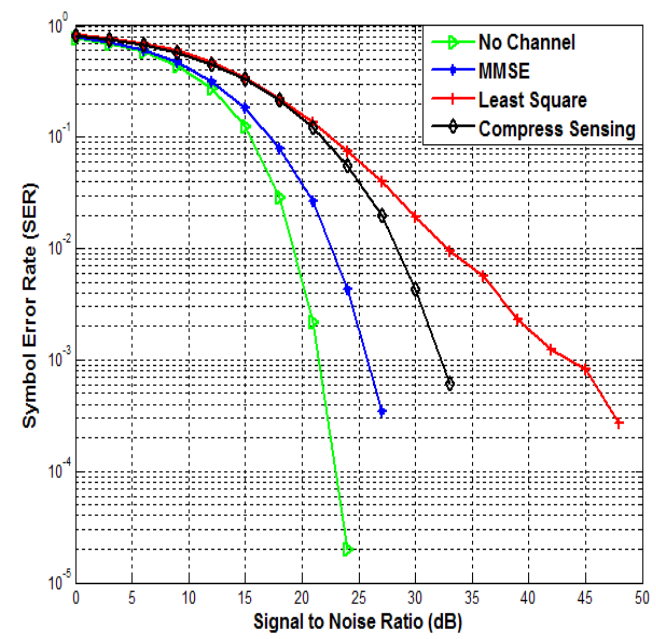

(d) 


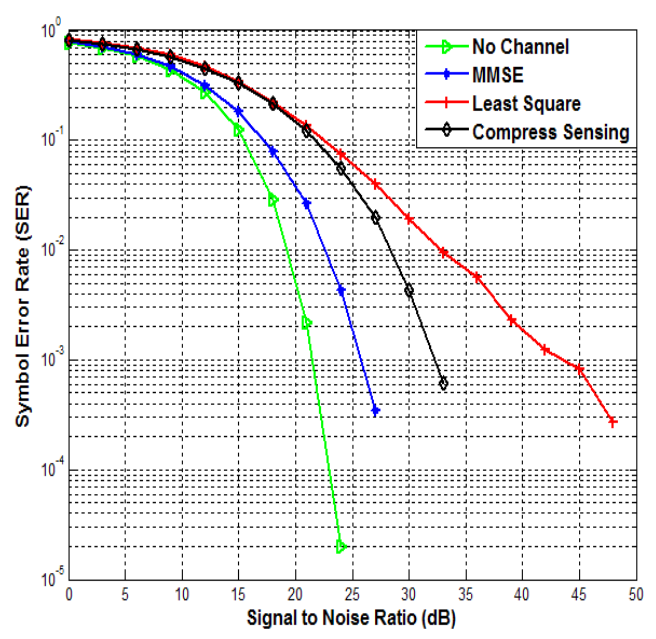

(e)

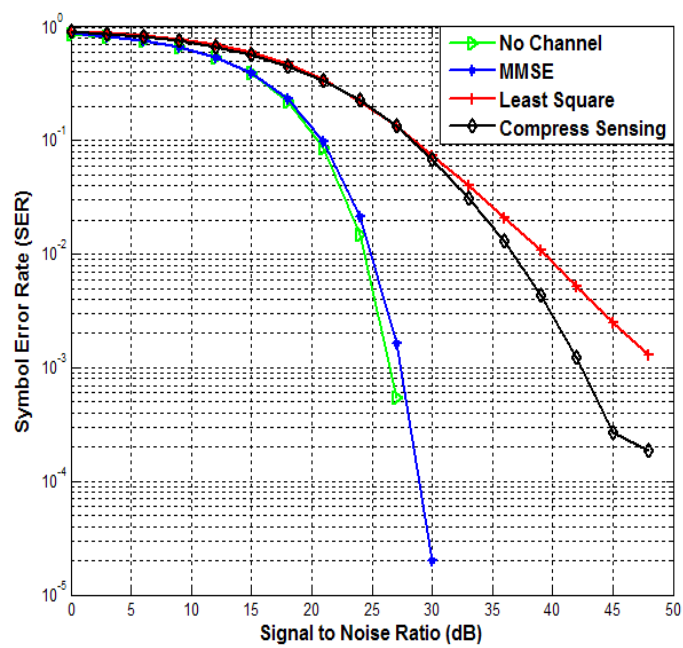

(f)

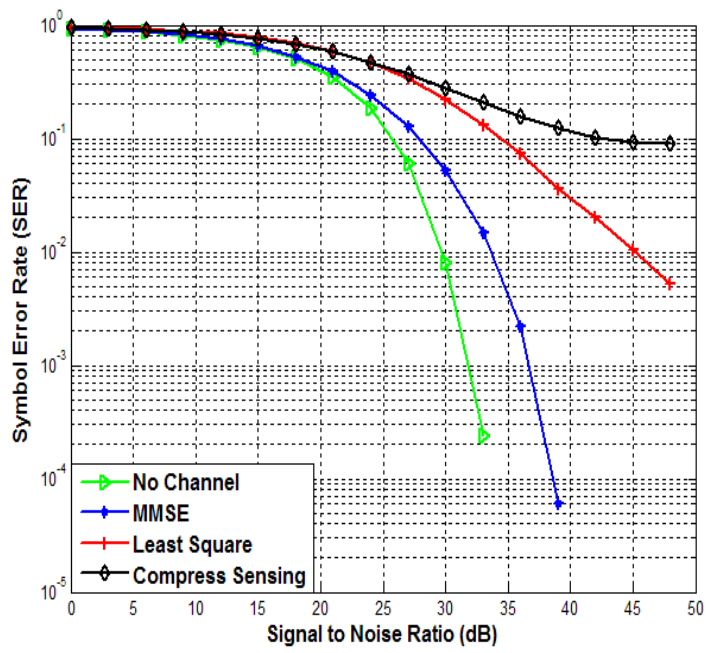

(g)

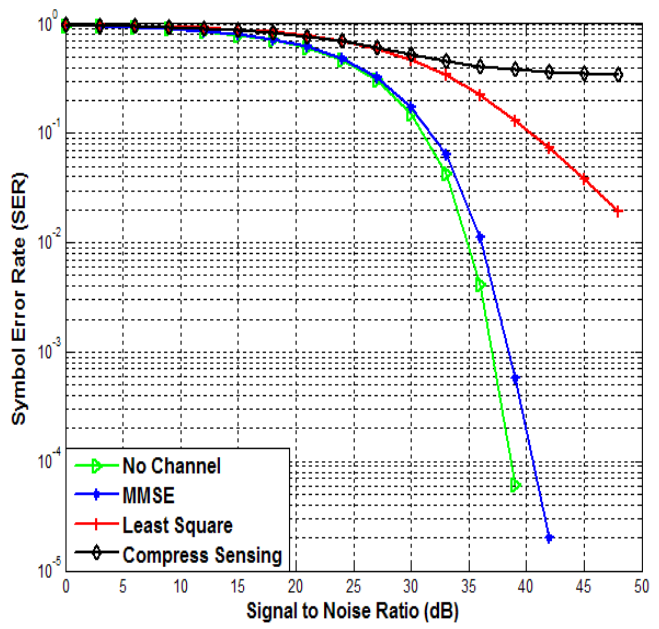

(h)

Figure 3 (a-h): SER vs SNR comparison for LS, MMSE and CS-DST estimating technique in Rician Channel (a) BPSK (b) QPSK (c) 8-PSK (d) 16-PSK (e) 32-PSK (f) 64-PSK (g) 128-PSK (h) 256-PSK.

\section{Conclusion}

In this paper, we have studied about the channel estimation schemes based on Compressed Sensing theory. Here Compressed Sensing is done in DST domain We firstly showed the performance evaluation of LS, MMSE and CS method based on M-PSK modulation. Based on channel statistics we made a comparison of the above schemes in the different channel. The performance of LS is worst among all the estimator used at both the channel as it does bot consider anything for noise reduction and interpolation. MMSE becomes the favourite among all the techniques with known CSI. As in practical channel information is much difficult to obtain we moved to CS estimation from which we infer CS is better at low SNR but as SNR increases it matches to LS estimation at higher order modulation. In future this can be implemented on QAM modulation and this approach can be tested for more iterations to reduce the complexity of the system. Delay factor can also be taken into consideration in future.

\section{References}

1. Cheng P, Chen Z, Rui Y. Channel estimation for OFDM systems over doubly selective channels: A distributed com- 
pressive sensing based approach. IEEE Transactions on Communications. 2013; 61:4173-85.

2. Xiong X, Jiang B, Gao X, You X. DFT-based channel estimator for OFDM systems with leakage estimation. IEEE Communications Letters. 2013; 17:1592-5.

3. Chen JC, Wen CK, Ting P. An efficient pilot design scheme for sparse channel estimation in OFDM systems. IEEE Communications Letters. 2013; 17:1352-5.

4. Tropp A, Gilbert AC. Signal recovery from random measurements via orthogonal matching pursuit. IEEE Transactions on Information Theory. 2007 Dec; 53(12):4655-66.

5. Cotter SF, Sparse channel estimation via matching pursuit with application to equalization. IEEE Communication Letter. 2002; 50:374-7.
6. Carbonelli C. Sparse channel estimation with zero tap detection. IEEE Transaction on Wireless Communication. 2007; 6:1743-63.

7. Bajwa WU. Compressed channel sensing: A new approach to estimating sparse multipath channels. IEEE Transaction on Vehicular Technology. 2010; 98:1058-76.

8. Qi C. Pilot design schemes for sparse channel estimation in OFDM systems. IEEE Transaction on Vehicular Technology. 2015; 64:1493-505.

9. Donoho DL. Uncertainty principles and ideal atomic decomposition. IEEE Transaction in Field Theory. 2001; 47:2845-62. 